\title{
In search of the allergen-driven chronic rhinosinusitis endotype
}

It is my pleasure to introduce the October 2021 issue of Rhinology, which promises novel insights into the pathophysiology of rhinologic disease, highlighting the important role of the sinonasal cavity - through which up to $95 \%$ of passively inhaled air first passes ${ }^{(1)}$ - as an important interface and barrier site for airborne environmental antigens and pathogens. In this issue of Rhinology, the article by Wee et al. is an epidemiologic study performed in a Korean national cohort of over 30,000 patients, which demonstrates an association between exposure to nitrogen dioxide air pollution and the development of chronic rhinosinusitis $(\mathrm{CRS})^{(2)}$. Another very interesting study by Hong et al demonstrates how the baseline inflammatory state of the sinonasal cavity may modulate susceptibility and responsiveness of the mucosal immune system to viral pathogens, with a specific focus on the receptor for the novel coronavirus that causes COVID-19(3). Giordano et al. demonstrate what many rhinologists have noticed in their practices during the COVID-19 pandemic - that filtration of airborne aeroallergens through the use of masks has been associated with a reduction in allergen-driven disease manifestations, such as allergic rhinitis symptoms ${ }^{(4)}$. These findings provide wonderful evidence that can be used to support recommendations for more common usage of face masks - for example during yard work in our pollen-sensitized patients - even after the pandemic has subsided.

The relationship between environmental aeroallergen hypersensitivity and CRS has been one that has received much attention and discussion over the years with increasing opinion that despite their common co-occurrence, aeroallergen hypersensitivity (and associated nasal allergy) modulates but is likely not the primary mechanism of development or persistence for CRS. So much distinction has been drawn between allergic rhinitis and CRS that a letter by Prof. Scadding, entitled "What's in a name - is CCAD really PPAR?"(5) in this issue of Rhinology cautions against referring to central compartment atopic disease (CCAD) - a putatively allergy-driven process characterized by polypoid inflammation of the middle turbinate and proximal aspects of the paranasal sinuses ${ }^{(6,7)}$ - as a subtype of CRS since this may lead to inappropriate disregard for effective allergy-specific treatments which have equivocal support as evidence-based treatments of CRS ${ }^{(6)}$. Although CCAD is defined phenotypically, the underlying concept is intriguing as an allergen-driven endotype of CRS. Does CCAD conceptually reflect a possible pathophysiological, mechanistic "missing link" between allergy and CRS? The phenotypic definition of CCAD is consistent with the physiological limitations to direct allergenstimulated sinus inflammation since airborne allergens would not be able to physically penetrate (and therefore cause an inflammatory response) deeper than the proximal aspects of the paranasal sinuses that are affected in CCAD. Furthermore, the descriptive entity of CCAD could open doors to the identification of a cellular- or molecularly-defined allergen-driven endotype of CRS as well as motivate clinical trials on the efficacy of anti-allergy treatments in this more specific population of CRS patients. Like the discussions surrounding all interesting and clinically meaningful questions, the pendulum swings back and forth regarding the relationship between the environment, allergy and CRS. I invite you to enjoy the latest scientific insights in this issue of Rhinology.

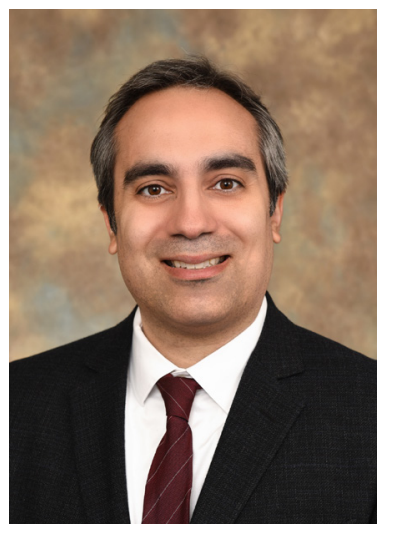

Ahmad R. Sedaghat, Associate Editor Cincinnati, $\mathrm{OH}$, USA

\section{References}

1. Fitzpatrick MF, Driver HS, Chatha N, Voduc N, Girard AM. Partitioning of inhaled ventilation between the nasal and oral routes during sleep in normal subjects. J Appl Physiol (1985) 2003; 94:883-890.

2. Wee JH, Min C, Jung HJ, Park MW, Park B, Choi HG. Association between air pollution and chronic rhinosinusitis: a nested casecontrol study using meteorological data and national health screening cohort data.
Rhinology 2020; 59(5):451-459.

3. Hong SN, Kim JK, Park JA, et al. Viral stimulation modulates endotype-related ACE2 expression in eosinophilic chronic rhinosinusitis. Rhinology 2020; 59(5): 460-469.

4. Liccardi G, Bilò MB, Milanese M, et al., Face masks during COVID-19 pandemic lockdown and self-reported seasonal allergic rhinitis symptoms. Rhinology 2020; 59(5): 481-484.

5. Scadding GK. What's in a name - is CCAD really PPAR? Rhinology 2020; 59(5):488.

6. Fokkens WJ, Lund VJ, Hopkins C, et al. European Position Paper on Rhinosinusitis and Nasal Polyps 2020. Rhinology 2020; 58:1-464.

7. Hamizan AW, Loftus PA, Alvarado R, et al Allergic phenotype of chronic rhinosinusitis based on radiologic pattern of disease. Laryngoscope. 2018;128(9):2015-2021. 